\title{
CARACTERÍSTICAS DE LA PESQUERÍA COMERCIAL EN LA CUENCA DEL RÍO UCAYALI
}

\author{
Edward L. ZORRILLA ${ }^{1}$, Antonia VELA², Paúl MURO ${ }^{1}$, Astrid DAÑINO² \\ 1 Universidad Nacional Intercultural de la Amazonía, Facultad de Ingeniería Agroforestal Acuícola. Ucayali, Perú. E-mail: \\ leni2787@gmail.com*. \\ 2 Instituto de Investigaciones de la Amazonía Peruana. Programa para el Uso y Conservación del Agua y sus Recursos- \\ AQUAREC, Sede Ucayali, Carretera Federico Basadre Km 12,400-Pucallpa (Perú).
}

\section{RESUMEN}

Para caracterizar la pesquería de consumo en la región Ucayali, se utilizó los datos recopilados en la zona de desembarque del Malecón Grau, en la ciudad de Pucallpa, durante los años 2011-2013. La información fue analizada dividiendo la cuenca del río Ucayali en zona alta y zona baja. Un total de 814 embarcaciones fueron identificadas, las cuales contribuyeron al desembarque de 8,419.64 toneladas de pescado. El mayor aporte fue registrado durante el periodo de vaciante del río Ucayali. Cuatro tipos de artes de pesca fueron identificadas en esta cuenca, siendo la más utilizada la red conocida regionalmente como "hondera". El desembarque pesquero estaba compuesto por 56 especies de peces registradas por sus nombres comerciales. La especie con mayor volumen de captura fue Prochilodus nigricans "boquichico", y representó el 36.46\% del volumen total registrado. El mayor índice de captura por unidad de esfuerzo (CPUE) fue obtenido en el bajo Ucayali con un total de $921.35 \mathrm{Kg}$. viaje $\mathrm{e}^{-1}$.

PALABRAS-CLAVES: Pesca comercial, Captura por unidad de esfuerzo, Río Ucayali.

\section{CHARACTERISTICS OF COMMERCIAL FISHERYES THAT OPERATE ON THE UCAYALI RIVER BASIN}

\begin{abstract}
To characterize Ucayali's regional fisheries, data compiled at the "Malecón Grau" unloading area were used, corresponding to the years 2011-2013. Information was analyzed by dividing areas into high and low Ucayali River. A total of 814 vessels were identified which contributed a total of 8419.864 tons of fish unloaded. The major contribution was recorded during the low water hydrological cycle. Four types of fishing techniques were identified and the net, also known regionally as "hondera", was the most commonly used. The capturing was compiled of 56 species recorded by their commercial names. The species with the greatest capture was Prochilodus nigricans "boquichico" representing $36.46 \%$ of the total fishery. The low Ucayali had the greatest value of catch per unit effort (CPUE) with a total of $921.35 \mathrm{~kg}$ trip-1.
\end{abstract}

KEYWORDS: Commercial fisheries, Catch per unit effort, Ucayali River. 


\section{INTRODUCCIÓN}

La cuenca amazónica comprende una superficie aproximada de 6'869,000 $\mathrm{Km}^{2}$ compartido principalmente por Brasil, Perú, Colombia, Ecuador, Bolivia, Venezuela y Guyana: La Amazonía peruana ocupa una extensión aproximada de 768,000 $\mathrm{Km}^{2}$ y representa el $60 \%$ del territorio nacional (Gallo, 1999). La ictiofauna amazónica es caracterizada por una riqueza de especies (Riofrio, 1998) y se estima entre 6000 a 8000 el número de especies ícticas que existen en la Amazonía, por lo que es considerado como el lugar de mayor diversidad de peces (Tello \& García, 2009).

La pesca en la Amazonía peruana es considerada como una de las principales actividades económicas de los centros poblados ubicados en la ribera de los ríos (Tello, 1995). El hombre amazónico construye sus viviendas cerca a los cursos de agua debido a que le permite contar con una vía de comunicación económica, así como disponer de alimento abundante y barato, como es el caso del pescado, principal abastecedor de proteína animal en la alimentación familiar (Tello, 1995). En la actividad pesquera de consumo es posible distinguir la pesca de subsistencia y la pesca comercial (Wasiw et al. (2012)), siendo la pesquería comercial una actividad extractiva y dinámica, lo cual se ha desarrollado sin una planificación adecuada (Riofrio, 1998).

Año a año se ha ejercido la actividad pesquera, por lo que se está viendo un mayor esfuerzo de pesca en la actualidad. En la región Ucayali la pesquería es una actividad importante por el papel que cumple en satisfacer la demanda de proteínas de la población y se hace necesario conocer los principales aspectos de la pesquería y la biología de los recursos más explotados en la región, con la finalidad de ejercer un adecuado manejo de los mismos y que no lleve al colapso a esta actividad (Riofrio et al., 2008) ni a la sobreexplotación del recurso (Riofrio, 1998).

En el presente estudio, se dan a conocer los resultados obtenidos del análisis de las estadísticas de captura y esfuerzo de pesca con base en la información disponible de los años 2011 - 2013 recolectados por el Instituto de Investigaciones de la Amazonía Peruana (IIAP), teniendo como finalidad caracterizar la pesquería de consumo que operan en el alto y bajo Ucayali, señalando la principales zonas de pesca, embarcaciones, artes de pesca, recursos explotados y la captura por unidad de esfuerzo.

\section{MATERIALES Y MÉTODOS}

El registro de información pesquera fue colectado por el Instituto de Investigaciones de la Amazonía Peruana (IIAP), en la zona de desembarque de "Malecón Grau", principal desembarcadero de la flota pesquera de Pucallpa, ubicado a $8^{\circ} 23^{\prime} 17.88^{\prime \prime}$ de latitud Sur y $74^{\circ} 31^{\prime} 42.09^{\prime \prime}$ de longitud Oeste, distrito de Callería, Provincia de Coronel Portillo.

A partir de estos registros se analizó la información recopilada de los años 2011 hasta 2013 procedentes de la cuenca del río Ucayali. Los datos de las fichas semiestructuradas, fueron agrupadas según la necesidad del análisis en: a) Producción pesquera en relación a la fluctuación del nivel del río, b) Principales zonas de pesca. Los datos fueron analizados agrupando la información en dos grandes zonas en esta cuenca: Alto Ucayali (AU) y Bajo Ucayali (BU). Fue considerado como Alto Ucayali desde el nacimiento del río Ucayali, ubicado a $10^{\circ} 41^{\prime}$ 26.06" latitud Sur y $73^{\circ} 47^{\prime} 27.40^{\prime \prime}$ latitud Oeste (unión con el río Tambo y Urubamba) hasta la zona de desembarque en Pucallpa ubicado a $8^{\circ} 23^{\prime} 17.88^{\prime \prime}$ de latitud Sur y $74^{\circ} 31^{\prime} 42.09$ " de longitud Oeste, incluyendo las diferentes zonas que existen en el transcurso del río y los diversos afluentes que desembocan en el río en dicha zona; fue considerado como Bajo Ucayali desde la zona de desembarque en Pucallpa hasta el registro de las diferentes zonas de pesca que pertenecen a la región de Loreto a $4^{\circ} 27^{\prime}$ 35.44" latitud Sur y $73^{\circ} 26^{\prime} 11.58^{\prime \prime}$ latitud Oeste (incluyendo a los diferentes afluentes que desembocan en el río Ucayali en esta zona). También fueron determinados cuales son las principales artes de pesca utilizadas, la Captura por Unidad de Esfuerzo (CPUE) y las principales especies de peces desembarcadas en la zona evaluada.

\section{RESULTADOS}

Durante los años de estudio la flota pesquera estaba compuesta por 814 embarcaciones que en conjunto aportaron un volumen de $8,419.864 \mathrm{t}$ al desembarque pesquero de esta parte del río Ucayali, el mayor desembarque fue registrado en el 2011 con un total de 3,039.595 t. Llevando en consideración el ciclo hidrológico, el máximo aporte fue registrado en la vaciante del año 2013, con un total de $526.525 \mathrm{t}$ y el mínimo en la creciente del 2012, con un total de $25.183 \mathrm{t}$ (Figura 01).

El desembarque pesquero para el alto Ucayali (AU) fue de 4,662.890 t y para el bajo Ucayali (BU) se reportó un total de 3,450.460 t. El 3.64\% restante fue aportado por embarcaciones que no tenían registros completos y que se le denominaron "SRC" ( $\sin$ registro completo), en las cuales no era posible registrar: tipo de artes de pesca, zonas de pesca, destino de pesca (tanto para el AU y BU), nombre de la embarcación y nombre del propietario. 


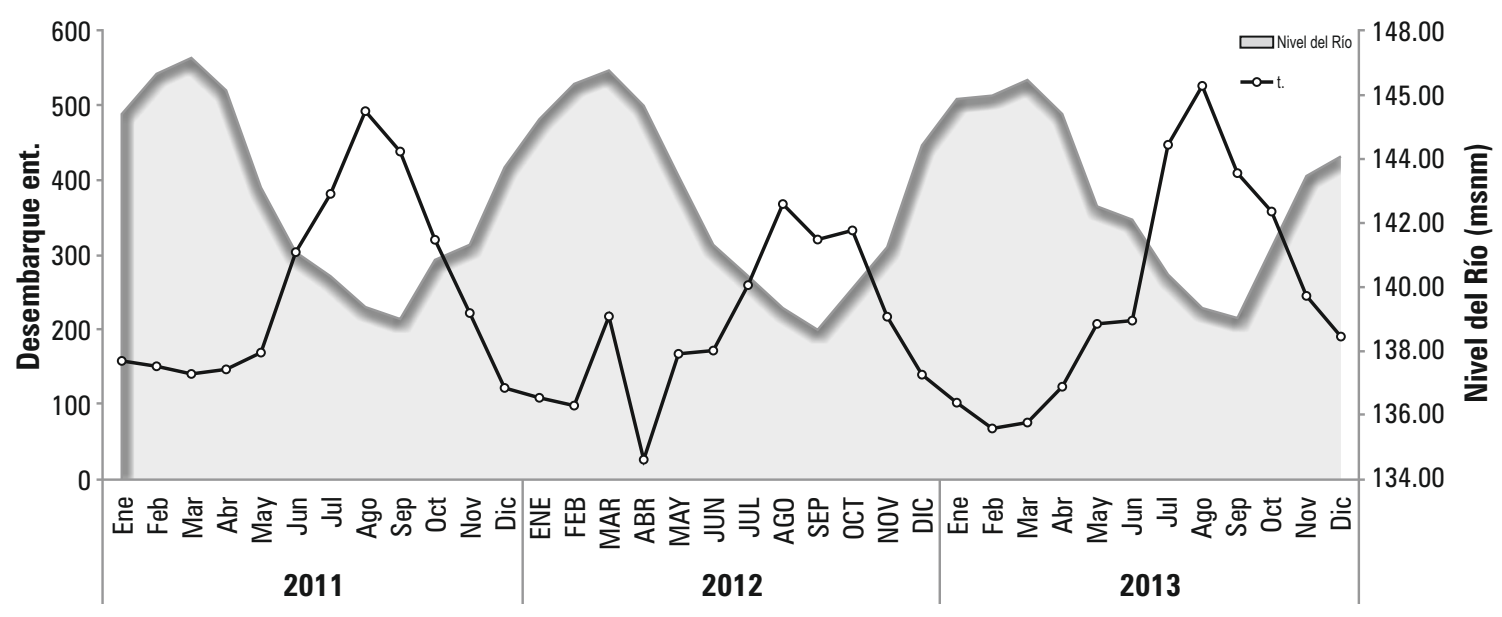

Figura 1. Desembarque pesquero comparado con el ciclo hidrológico.

Fueron registrados un total de 225 zonas de pesca, frecuentados por la flota pesquera de Pucallpa. El 53.77\% de estas zonas (121), fueron registrados para el alto Ucayali; las zonas más sobresalientes en esta área fueron: Runuya (13.28\%), Nuevo Italia (7.57\%), Chauya (7.36\%), Utucuro (6.09\%), Agua Negra (5.88\%) y Pachitea (4.6\%). En la zona del bajo Ucayali fue registrado el $46.33 \%$ (104 zonas) de las zonas restantes. En esta parte del río Ucayali los mayores aportes al desembarque pesquero provinieron de Contamana $(8.14 \%)$, seguida por Shapajal $(7.35 \%)$, Orellana $(6.36 \%)$, Tiruntan $(5.92 \%)$, Calleria $(5.28 \%)$ y Tacshitea $(5.23 \%)$.

Cuando los datos de desembarque pesquero fueron agrupados por años, observamos que en el 2012 la pesca abarco un mayor número de zonas que en el 2013 (160 y 143 zonas, respectivamente), siendo que las principales zonas de pesca se ubicó cerca de la zona del desembarque pesquero de Pucallpa.

En el periodo evaluado, las artes de pesca (Figura 02) más utilizadas tanto en el alto como en el bajo Ucayali fueron la hondera $(62.98 \%$ y $75.41 \%$ respectivamente), seguidas por la red trampera (28.51\% y $13.95 \%$ respectivamente) y red rastrera ( $7.37 \%$ y $7.27 \%$ respectivamente), el anzuelo fue un arte de pesca muy poco utilizado en el periodo evaluado, presentando porcentajes menores al $1 \%$ del total $(0.79 \%$ y $0.50 \%$ respectivamente $)$. El porcentaje de artes de pesca no reportado por las embarcaciones "SRC" fue menor en el Alto Ucayali que en bajo Ucayali $(0.34 \%$ y $2.87 \%$, respectivamente).

En consideración a la información disponible de desembarque y esfuerzo de pesca de la flota pesquera de Pucallpa (2011-2013) se determinó que el bajo Ucayali obtuvo un mayor índice de CPUE $\left(921.35 \mathrm{Kg}\right.$. viaje $\left.{ }^{-1}\right)$ a diferencia del alto Ucayali (709.51 Kg. viaje ${ }^{-1}$ ) (Cuadro 01). Indicando que al realizar un viaje en las diferentes zonas del bajo Ucayali se puede extraer $921.35 \mathrm{~kg}$ de pescado.

La diversidad de peces extraídos para el consumo fue alta, tanto para el alto y bajo Ucayali, identificándose durante el estudio a 56 especies basados en su nombre común o nombres comerciales, abarcando muchos de ellos a varias especies en un mismo nombre (cuadro 02), de las cuales diez de ellas representaron el $87.67 \%$ del desembarque total destacando las principales y de mayor aporte como el Prochilodus nigricans "Boquichico" (36.46\%), Pimelodus sp. "Bagre" (18.25\%), Triportheus angulatus y T. elongatus "Sardina" (7.96), Mylossoma duriventris "Palometa" (6.49\%), Potamorhina altamazonica "Llambina" (5.27\%), Schizodon spp. y Leporinus spp "Liza" (4.08\%), Calophysus macropterus "Mota" (3.52\%), Pseudoplatystoma sp y P. punctifer "Doncella" (2.16\%), Psectrogaster rutiloides "Chio chio" (1.92\%) y Serrasalmus spp, Pygocentrus nattereri "Paña" $(1.55 \%)$.

\section{DISCUSIÓN}

Wasiw et al. (2012), menciona que durante los años 1992 a 1998 las zonas de pesca se ubicaban cerca de los lugares de desembarque, pero en el 2010 fueron más distantes y muchas de ellas estuvieron fuera de la región de Ucayali coincidiendo con el análisis del presente estudio, reportando a Contamana (región Loreto) como una las principales zonas que más aportó al desembarque pesquero del bajo Ucayali. 


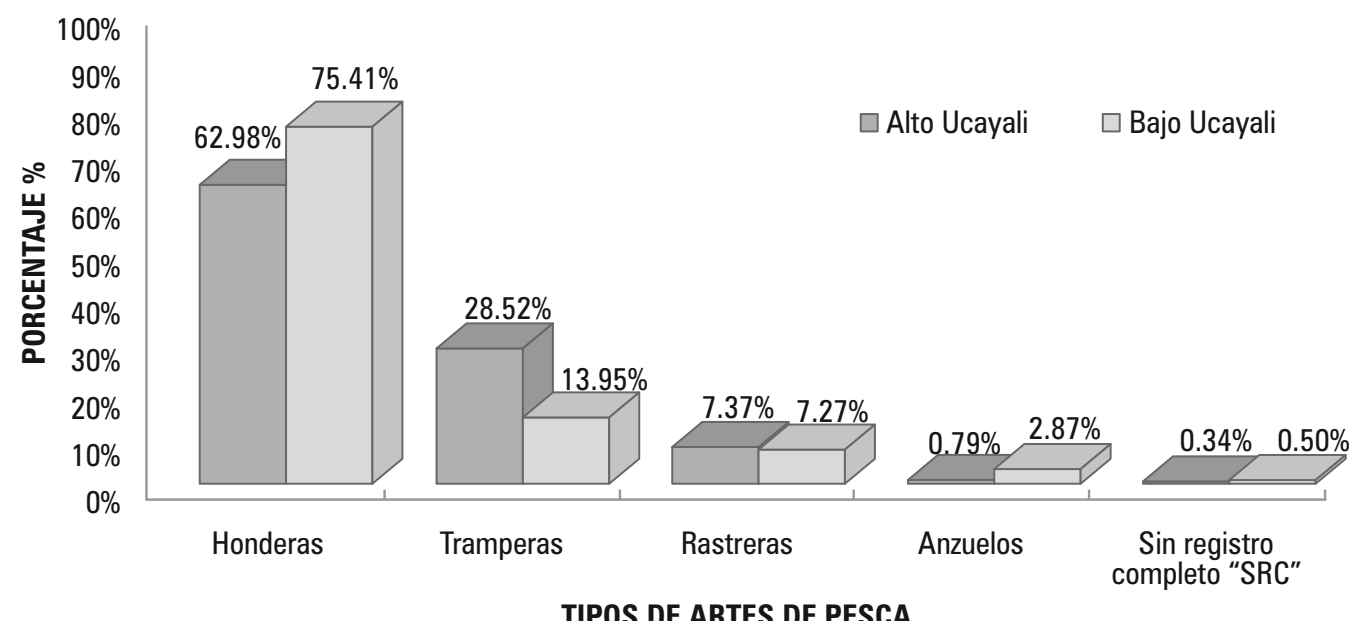

Figura 2. Artes de pesca utilizada en el alto y bajo Ucayali.

Autores como Guerra (1995), Tello (1995), Riofrio (1998), Tello \& Bayley (2001), Álvarez \& Ríos (2008) y Wasiw et al. (2012) mencionan en sus resultados las mismas artes de pesca tales como la hondera, rastrera, trampera y anzuelo, que son frecuentemente utilizados por la flota comercial de Pucallpa y de Iquitos.

Es necesario señalar que en las últimas décadas la pesca ha sufrido importantes modificaciones, debido a la inclusión de fibra sintética, permitiendo capturas masivas, sobretodo de la red denominado "hondera", que es la red más representativa de las capturas comerciales en la zona del presente estudio.

Cabrera \& Salas (1999) mencionan que para fines de manejo de una pesquería, es fundamental tener una medida real del esfuerzo pesquero aplicado sobre el recurso explotado; Pero cuando se trata de pesquerías artesanales en áreas tropicales el problema se hace complejo, dado que existe diversidad de artes y métodos de pesca, así como cambios estacionales en la especie objetivo. Durante el presente estudio se obtuvo que el mayor aporte en el índice CPUE fue del bajo Ucayali $\left(921.35 \mathrm{Kg}\right.$. viaje $\left.\mathrm{e}^{-1}\right)$ siendo menor a lo reportado por Riofrio (1998) indicando que la flota congelera para Ucayali es de 3'684 kg. viaje ${ }^{-1}$

El estado de los recursos pesqueros continentales es muy dinámico y están siendo afectados por la actividad antropogénica, presentando síntomas de presión de pesca en algunas especies, existiendo un reemplazo de las especies de gran tamaño por especies más productivas y pequeñas (Tello \& Bayley 2001), afirmándose que hace 17 años las principales especies capturadas estaban compuestas por Prochilodus nigricans "Boquichico", Brachyplatystoma rousseauxii "Dorado", Colossoma macropomum "Gamitana" Pseudoplatystoma fasciatum "Doncella", Piaractus brachypomus "Paco", Potamorhina altamazónica "Llambina", Hypophthalmus spp "Maparate", Mylossoma duriventris "Palometa", Arapaima gigas "Paiche" y Plagioscion spp "Corvina" (Riofrio,1998) diferenciándose a lo reportado durante el presente estudio.

Tabla 1. Captura Por Unidad de Esfuerzo en el Alto y Bajo Ucayali del 2011-2013.

\begin{tabular}{ccccc}
\hline $\begin{array}{c}\text { Alto y Bajo } \\
\text { Ucayali }\end{array}$ & Cantidad (t) & \# De Viajes & $\begin{array}{c}\text { CPUE } \\
\text { (Kg. viaje-1). }\end{array}$ & $\begin{array}{c}\text { \% De Captura } \\
\text { (t) }\end{array}$ \\
\hline Alto Ucayali & 4662.890 & 6572 & 709.51 & 55.38 \\
Bajo Ucayali & 3450.460 & 3745 & 921.35 & 40.98 \\
\hline
\end{tabular}


Tabla 2. Lista de especies capturadas por la flota pesquera de Pucallpa del año 2011-2013.

\begin{tabular}{|c|c|c|c|c|c|c|}
\hline $\begin{array}{l}\text { Nombre } \\
\text { Científico }\end{array}$ & $\begin{array}{l}\text { Nombre } \\
\text { Comercial }\end{array}$ & $\begin{array}{c}A U . \\
\text { (Cap. en t) }\end{array}$ & $\begin{array}{c}\text { BU. } \\
\text { (Cap. en t) }\end{array}$ & $\begin{array}{c}\text { "SRC". } \\
\text { (Cap. en t) }\end{array}$ & TOTAL (t) & $\%$ \\
\hline Prochilodus nigricans & Boquichico & $1793.42 \pm 53.58$ & $1246.98 \pm 47.43$ & $29.57 \pm 1.48$ & $3069.97 \pm 47.42$ & 36.46 \\
\hline Pimelodus spp & Bagre & $853.95 \pm 34.91$ & $668.22 \pm 34.22$ & $14.38 \pm 1.89$ & $1536.55 \pm 32.16$ & 18.25 \\
\hline $\begin{array}{l}\text { Triportheus angulatus, } \\
\text { Triportheus elongatus }\end{array}$ & Sardina & $285.23 \pm 7.12$ & $379.08 \pm 11.79$ & $9.07 \pm 0.52$ & $673.38 \pm 9.45$ & 8.00 \\
\hline Mylossoma duriventris & Palometa & $244.12 \pm 8.25$ & $277.48 \pm 11.39$ & $24.71 \pm 1.08$ & $546.30 \pm 8.98$ & 6.49 \\
\hline Potamorhina altamazonica & Llambina & $336.80 \pm 13.82$ & $102.70 \pm 7.50$ & $4.55 \pm 0.67$ & $444.05 \pm 11.41$ & 5.27 \\
\hline $\begin{array}{l}\text { Schizodon spp, } \\
\text { Leporinus spp, } \\
\text { Rythiodus spp, } \\
\text { Pseudanos spp }\end{array}$ & Liza & $198.36 \pm 5.11$ & $139.97 \pm 6.29$ & $7.93 \pm 0.60$ & $346.25 \pm 5.42$ & 4.11 \\
\hline Calophysus macropterus & Mota & $80.11 \pm 1.43$ & $202.92 \pm 3.99$ & $13.66 \pm 0.46$ & $296.69 \pm 3.31$ & 3.52 \\
\hline $\begin{array}{l}\text { Pseudoplatystoma punctifer, } \\
\text { Pseudoplatystoma sp. }\end{array}$ & Doncella & $60.04 \pm 1.02$ & $49.77 \pm 1.61$ & $71.91 \pm 1.59$ & $181.72 \pm 1.48$ & 2.16 \\
\hline Psectrogaster rutiloides & Chiu-Chiu & $81.01 \pm 6.00$ & $77.56 \pm 5.25$ & $2.95 \pm 0.36$ & $161.52 \pm 5.20$ & 1.92 \\
\hline $\begin{array}{c}\text { Serrasalmus spp, } \\
\text { Pygocentrus nattereri }\end{array}$ & Paña & $106.66 \pm 2.89$ & $21.75 \pm 0.60$ & $2.41 \pm 0.09$ & $130.82 \pm 2.27$ & 1.55 \\
\hline $\begin{array}{l}\text { Rhaphiodon vulpinus, } \\
\text { Hydrolycus scomberoides }\end{array}$ & Chambira & $87.54 \pm 2.70$ & $26.87 \pm 0.78$ & $1.99 \pm 0.09$ & $116.40 \pm 2.03$ & 1.38 \\
\hline $\begin{array}{l}\text { Hypophthalmus edentatus, } \\
\text { Hypophthalmus marginatus }\end{array}$ & Maparate & $88.20 \pm 196$ & $21.72 \pm 0.71$ & $3.07 \pm 0.08$ & $112.99 \pm 1.66$ & 1.34 \\
\hline $\begin{array}{c}\text { Plagioscion squamosissimus, } \\
\text { Plagioscion auratus }\end{array}$ & Corvina & $64.71 \pm 1.92$ & $27.53 \pm 0.87$ & $11.76 \pm 0.61$ & $104.00 \pm 1.44$ & 1.24 \\
\hline Hoplias malabaricus & Fasaco & $82.84 \pm 2.37$ & $18.67 \pm 0.56$ & $2.36 \pm 0.08$ & $103.87 \pm 1.83$ & 1.23 \\
\hline Sorubim lima & Shiripira & $52.48 \pm 1.80$ & $34.10 \pm 1.57$ & $2.20 \pm 0.12$ & $88.78 \pm 1.61$ & 1.05 \\
\hline $\begin{array}{l}\text { Anodus elongatus, } \\
\text { Hemiodus sp }\end{array}$ & Yulilla & $50.47 \pm 1.96$ & $12.73 \pm 0.85$ & $0.25 \pm 0.03$ & $63.45 \pm 1.68$ & 0.75 \\
\hline $\begin{array}{l}\text { Liposarcus spp, } \\
\text { Glyptoperichthys spp, } \\
\text { Hypostomus spp }\end{array}$ & Carachama & $16.57 \pm 0.56$ & $12.04 \pm 0.90$ & $25.97 \pm 0.66$ & $54.58 \pm 0.74$ & 0.65 \\
\hline Megaladoras irwini & Piro & $26.89 \pm 0.94$ & $9.63 \pm 0.36$ & $15.89 \pm 0.81$ & $52.40 \pm 0.77$ & 0.62 \\
\hline Epapterus dispilurus & Leguía & $33.6 \pm 2.6$ & $10.15 \pm 1.05$ & $0.15 \pm 0.04$ & $43.90 \pm 1.81$ & 0.52 \\
\hline Psectrogaster amazónica & Ractacara & $19.03 \pm 3.10$ & $15.38 \pm 1.82$ & $3.26 \pm 0.48$ & $37.67 \pm 2.38$ & 0.45 \\
\hline $\begin{array}{l}\text { Brycon melanopterus, } \\
\text { Brycon erythropterum, }\end{array}$ & Sábalo & $12.49 \pm 1.23$ & $18.14 \pm 1.04$ & $0.67 \pm 0.05$ & $31.31 \pm 1.07$ & 0.37 \\
\hline $\begin{array}{l}\text { Ageneiosus ucayalensis, } \\
\text { Ageneiosus brevifilis }\end{array}$ & Bocon & $9.36 \pm 0.41$ & $8.28 \pm 0.34$ & $2.21 \pm 0.15$ & $19.85 \pm 0.35$ & 0.24 \\
\hline Brachyplatystoma juruense & Achune & $3.32 \pm 0.12$ & $6.58 \pm 0.30$ & $7.20 \pm 0.31$ & $17.10 \pm 0.27$ & 0.20 \\
\hline Piaractus brachypomus & Paco & $1.81 \pm 0.12$ & $5.72 \pm 0.35$ & $5.77 \pm 0.31$ & $13.30 \pm 0.30$ & 0.16 \\
\hline Brachyplatystoma rousseauxii & Dorado & $2.26 \pm 0.09$ & $2.74 \pm 0.14$ & $8.49 \pm 0.21$ & $13.49 \pm 0.20$ & 0.16 \\
\hline
\end{tabular}


Continuación Tabla 2

\begin{tabular}{|c|c|c|c|c|c|c|}
\hline $\begin{array}{l}\text { Nombre } \\
\text { Científico }\end{array}$ & $\begin{array}{l}\text { Nombre } \\
\text { Comercial }\end{array}$ & $\begin{array}{c}\text { AU. } \\
\text { (Cap. en t) }\end{array}$ & $\begin{array}{c}\text { BU. } \\
\text { (Cap. en t) }\end{array}$ & $\begin{array}{c}\text { "SRC". } \\
\text { (Cap. en t) }\end{array}$ & TOTAL (t) & $\%$ \\
\hline $\begin{array}{l}\text { Satanoperca spp, } \\
\text { Cichlasoma spp, } \\
\text { Aequidens spp. }\end{array}$ & Bujurqui & $11.81 \pm 0.99$ & $0.70 \pm 0.15$ & 0.12 & $12.63 \pm 0.89$ & 0.15 \\
\hline Parauchenipterus galeatus & Novia & $3.14 \pm 0.11$ & $8.38 \pm 0.73$ & $0.39 \pm 0.03$ & $11.92 \pm 0.51$ & 0.14 \\
\hline Paulicea lutkeni & Llausa & $2.27 \pm 0.09$ & $3.93 \pm 0.16$ & $5.56 \pm 0.19$ & $11.76 \pm 0.17$ & 0.14 \\
\hline Brachyplatystoma vaillantii & Manitoa & $2,65 \pm 0.06$ & $8.82 \pm 2.37$ & 0.10 & $11.57 \pm 2.00$ & 0.14 \\
\hline Cichla monoculus & Tucunare & $7.35 \pm 0.29$ & $1.29 \pm 0.12$ & $2.90 \pm 0.12$ & $11.54 \pm 0.23$ & 0.14 \\
\hline Leiarius marmoratus & Ashara & $3.89 \pm 0.16$ & $5.29 \pm 0.25$ & $2.12 \pm 0.18$ & $11.30 \pm 0.20$ & 0.13 \\
\hline Phractocephalus hemioliopterus & Torre & $1.87 \pm 0.06$ & $4.84 \pm 0.23$ & $4.12 \pm 0.18$ & $10.83 \pm 0.18$ & 0.13 \\
\hline Brachyplatystoma platynemum & Vaselina & $2.64 \pm 0.11$ & $3.94 \pm 0.25$ & $4.25 \pm 0.18$ & $10.83 \pm 0.19$ & 0.13 \\
\hline Astronotus ocellatus & Acarahuazu & $4.46 \pm 0.25$ & $1.24 \pm 0.07$ & $5.05 \pm 0.30$ & $10.75 \pm 0.25$ & 0.13 \\
\hline Pellona castelnaeana & Panshin & $5.94 \pm 0.22$ & $1.62 \pm 0.09$ & $0.48 \pm 0.21$ & $8.04 \pm 0.19$ & 0.10 \\
\hline $\begin{array}{c}\text { Roeboides myersii, } \\
\text { Roeboides affinis, } \\
\text { Cynopotamus amazonus }\end{array}$ & Dentón & $4.25 \pm 0.43$ & $2.87 \pm 0.29$ & 0.20 & $7.32 \pm 0.36$ & 0.09 \\
\hline Pseudoplatystoma tigrinum Z & Zúngaro Tigre & $2.305 \pm 0.10$ & $1.45 \pm 0.08$ & $1.70 \pm 0.57$ & $5.45 \pm 0.09$ & 0.06 \\
\hline Pseudodoras niger & Turushuqui & $4.54 \pm 0.21$ & $0.30 \pm 0.03$ & $0.23 \pm 0.06$ & $5.07 \pm 0.19$ & 0.06 \\
\hline Hoplosternum littorale & Shirui & $3.95 \pm 0.31$ & $0.78 \pm 0.16$ & & $4.73 \pm 0.27$ & 0.06 \\
\hline Hoplerythrinus unitaeniatus & Shuyo & $1.84 \pm 0.14$ & $1.86 \pm 0.18$ & $0.35 \pm 0.03$ & $4.04 \pm 0.15$ & 0.05 \\
\hline Colossoma macropomum & Gamitana & $0.5 \pm 0.03$ & $1.22 \pm 0.09$ & $1.70 \pm 0.08$ & $3.42 \pm 0.08$ & 0.04 \\
\hline Sorubimichthys planiceps & Achacubo & $1.05 \pm 0.04$ & $1.59 \pm 0.11$ & $0.52 \pm 0.02$ & $3.17 \pm 0.07$ & 0.04 \\
\hline $\begin{array}{c}\text { Tetragonopterus, } \\
\text { Ctenobrycon }\end{array}$ & Mojara & $2.50 \pm 0.71$ & $0.49 \pm 0.05$ & & $2.99 \pm 0.50$ & 0.04 \\
\hline Arapaima gigas & Paiche & 0.3 & 0.09 & $1.77 \pm 0.20$ & $2.16 \pm 0.17$ & 0.03 \\
\hline Cynodon gibbus & Huapeta & $1.06 \pm 0.06$ & $1.08 \pm 0.07$ & & $2.14 \pm 0.06$ & 0.03 \\
\hline Brachyplatystoma filamentosum & Salton & $0.12 \pm 0.06$ & $0.50 \pm 0.08$ & $1.20 \pm 0.10$ & $1.82 \pm 0.09$ & 0.02 \\
\hline Hemisorubim platyrhynchos & Toa & $0.89 \pm 0.04$ & $0.67 \pm 0.06$ & & $1.56 \pm 0.05$ & 0.02 \\
\hline Potamotrygon spp & Raya & $0.19 \pm 0.01$ & $0.13 \pm 0.01$ & $0.97 \pm 0.05$ & $1.29 \pm 0.05$ & 0.02 \\
\hline Curimatella meyeri & San Pedro & $0.75 \pm 0.07$ & 0.03 & 0.06 & $0.84 \pm 0.07$ & 0.01 \\
\hline Osteoglossum bicirrhosum & Arahuana & $0.31 \pm 0.02$ & $1.6 \pm 0.04$ & $0.26 \pm 0.04$ & $0.72 \pm 0.03$ & 0.01 \\
\hline $\begin{array}{c}\text { Farlowella spp, } \\
\text { Sturisoma spp, } \\
\text { Loricaria sp, } \\
\text { Loricariichthys spp }\end{array}$ & Shitari & $0.49 \pm 0.05$ & $0.05 \pm 0.01$ & & $0.54 \pm 0.05$ & 0.01 \\
\hline Myleus rubripinnis & Curuhuara & $0.4 \pm 0.14$ & 0.03 & 0.03 & $0.45 \pm 0.13$ & 0.01 \\
\hline Brachyplatystoma sp & Filiote & 0.05 & $0.19 \pm 0.04$ & $0.06 \pm 0.03$ & $0.30 \pm 0.04$ & 0.004 \\
\hline Lycengraulis batesii & Chullacho & & 0.30 & & 0.30 & 0.004 \\
\hline Acestrorhynchus spp & Pez Zorro & $0.13 \pm 0.05$ & & & $0.13 \pm 0.05$ & 0.002 \\
\hline Pterodoras granulosus & Cahuara & 0.04 & 0.02 & & $0.06 \pm 0.01$ & 0.001 \\
\hline
\end{tabular}




\section{CONCLUSIÓN}

Se determinó que las características de la pesquería comercial en la zona de desembarque de Pucallpa, que actuaron tanto para el alto y bajo Ucayali, fueron diferentes; teniendo en cuenta que los mayores desembarques ocurrieron durante las vaciantes y las menores en crecientes; siendo la máxima aportación para el desembarque en el año 2013 con un total de 526.525 t y la mínima fue en el 2012 con $26.183 \mathrm{t}$.

Se registró 121 zonas de pesca pertenecieron al alto Ucayali, entre las que destacan: Runuya, Nuevo Italia, Chauya, Utucuro, Agua Negra y Pachitea; mientras que para el bajo Ucayali el número fue menor, registrando 104 zonas, tales como: Contamana, Shapajal, Orellana, Tiruntan, Calleria y Tacshitea.

El mayor registro de zonas fue para el año 2012 que obtuvo 160 de zonas, y la de menor fue en el año 2013 con 143 zonas, dado que para el año antes mencionado los mijanos de peces estaban frecuentemente cercanos y algunas embarcaciones no optaron ir muy lejos para realizar su actividad pesquera y las diferentes zonas del alto Ucayali durante este periodo fueron las principales para el desembarque pesquero tales como Runuya, Chauya y Nuevo Italia.

Las principales artes de pesca que actuaron para el alto y bajo Ucayali fueron: la "hondera" con $65.96 \%$, la "trampera" con $21.80 \%$, la "rastrera" con $7.05 \%$ y el "anzuelo" con $0.75 \%$. Siendo para ambos casos y de mayor aporte al desembarque pesquero la red denominada "hondera".

Se determinó a 56 especies extraídas por la flota pesquera comercial tanto para el alto y bajo Ucayali; no obstante se debe considerar que un nombre comercial puede referirse a más de una especie involucrando en algunos casos entre familias.

La mayor CPUE fue para el bajo Ucayali, con un índice de $921.35 \mathrm{Kg}$. viaje $^{-1}$; a diferencia del alto Ucayali que fue menor con un valor de $709.51 \mathrm{Kg}$. viaje- $^{1}$.

\section{BIBLIOGRAFÍA CITADA}

Alvarez, L. F.; Rios, S. J. 2008. Viabilidad económica de la pesca artesanal en el departamento de Loreto. (www.promamazonia. org.pe/wfr_Publicacion.aspx). Acceso: 21/10/16
Cabrera-Pérez, J. L.; Salas-Marquez, S. 1999. Análisis del esfuerzo pesquero en la pesquería de Langosta (Panulirus argus) en tres puertos de la península de Yucatán. In: Proceedings of the $45^{\text {th }}$ Gulf and Caribbean Fisheries Institute, p. 790808.

Gallo, R. 1999. Situación del manejo de las pesquerías de grandes bagres migratorios amazónicos en el Perú. In: FAO. Informe del Taller Regional sobre Manejo de las Pesquerías de Bagres Migratorios de la Amazonía, p. 79100 .

Guerra, H. 1995. Estado actual del conocimiento de la pesquería en la Amazonía Peruana. Instituto de Investigaciones de la Amazonía Peruana, Iquitos, $54 \mathrm{pp}$.

Riofrio, J. C. 1998. Características de la pesquería comercial de consumo en Pucallpa (UcayaliPerú). Revistas de Investigaciones Pecuarias, 9(1): 67-7.

Riofrio, J. C.; Ferré, W. R.; Velarde, D. A. 2008. Contribuciones para el manejo de la pesquería comercial en Pucallpa (Ucayali, Perú). In: Pinedo, D.; Soria, C. (Eds). El manejo de las pesquerías en ríos tropicales de Sudamérica. $p$. 205-234.

Tello, S. 1995. Relevamiento de información sobre captura y esfuerzo pesquero con destino a ciudades. Instituto de Investigaciones de la Amazonía Peruana, Iquitos, 60pp.

Tello, S., Bayley, P. 2001. La pesquería comercial de Loreto con énfasis en el análisis de la relación entre captura y esfuerzo pesquero de la flota comercial de Iquitos, cuenca del Amazonas (Perú). Folia Amazónica, 12(1-2):123-139.

Tello, S.; García, A. 2009. La pesquería de grandes bagres en la región Loreto. Instituto de Investigaciones de la Amazonía Peruana, Iquitos, 28pp.

Wasiw, J.; Riofrio, J.; Muñoz, V. 2012. Monitoreo de la pesquería comercial en Pucallpa y Yarinacocha 2010 (Ucayali - Perú). Informe IMARPE, 39(3-4): 288-293.

Recibido: 1 de Agosto del 2016

Aceptado para publicación: 30 de Agosto del 2016 
